\title{
Relationship between state anxiety and success rate in game performance, coach's evaluation among Japanese university volleyball players
}

\author{
Kai Yamada ${ }^{a *}$, Yujiro Kawata ${ }^{\mathrm{b}}$, Nobuyuki Nakajima ${ }^{\mathrm{a}, \mathrm{c}}$ and Masataka Hirosawa, ${ }^{\mathrm{a}, \mathrm{c}}$ \\ a Juntendo University Graduate School of Health and Sports Science, Chiba, Japan \\ ${ }^{\mathrm{b}}$ Tokyo Future University, Department of Child Psychology, Tokyo, Japan \\ ' Juntendo University School of Health and Sports Science, Chiba, Japan
}

\begin{abstract}
The purpose of this study is to examine the relationship between state anxiety and individual athletic game performance. The subjects were 54 male players of 4 teams (The average age was 20.5 ( $\mathrm{SD}=1.09$ ) years), who participated in the Kanto university men's volleyball league games in the spring of year 2010. The subjects were asked to respond to a Japanese version of the State Trait Anxiety Inventory (STAI), which was measured at 4 time periods (the day before, the day of game, just before the game, just after the game) for each of a total of 40 games (each team played 10 games). The results were as follows: (1) Relationships between state anxiety and success rate in game performance (spike, reception, block) showed different correlation depending on each game performance. (2) The group of players who performed well in games collected by coaches scored lower than the more badly-performing group in the score of state anxiety on the day of game and just after the game. (3) The temporal change of the score of state anxiety from just before the game to just after the game was different depending on whether they performed well or not in games.
\end{abstract}

Keywords: State anxiety, Success rate, Coach's evaluation, Individual performance, Volleyball

\section{Introduction}

State Trait Anxiety Inventory (STAI) is an instrument for measuring the level of state anxiety being held. The original version of STAI was established in 1970 by Spielberger et al [4]. It became easy to measure state anxiety even in sports athletes or players in the sports field and, since then, this relationship between state anxiety and game performance has been examined in a lot of studies using the STAI.

However, the majority of these studies examined the relationship between state anxiety and the team performance (the game results, for example) whilst few studies examined the relationship between state anxiety and individual performance such as the success rate in game performance and the coach's evaluation for the performance taking into consideration their true ability $[1,2]$.
Therefore, the purpose of this study is (1) to examine the relationship between state anxiety and the success rate in game performance (spike, reception, and block) among Japanese university volleyball players, and (2) to examine the relationship between state anxiety and the coach's evaluation for the performance taking into consideration the player's true ability.

\section{Methods}

We measured the following 3 indices in order to examine the above:

(1) State Anxiety: A questionnaire investigation was carried out on 54 Japanese male players of 4 teams, who participated in the Kanto university men's volleyball league games in the spring of year

\footnotetext{
*Corresponding author. E-mail: hitogahitowoerabu12@hotmail.co.jp
} 
2010. The average age of the subjects was 20.5 $(\mathrm{SD}=1.09)$ years. A Japanese translation of the version of State Trait Anxiety Inventory [3] was used in this study. This questionnaire was composed of 20 items, and the subjects were asked to respond to each item using the 4-point Likert scale. The total was the sum of the scores of 20 items (the score range $=20$ to 80 ). It was measured at 4 time periods (the day before, the day of game, just before the game, just after the game) for each of a total of 40 games (each team played 10 games).

(2) Success rate in game performance: The subjects were 49 players who played spike, reception, and block even once in the Kanto university men's volleyball league games in the spring of year 2010 . The average age of subjects was $20.6(\mathrm{SD}=0.96)$ years. Forty games were recorded, and the success rate of spike, reception, and block were calculated based on the Japan Volleyball Information System (JVIS) after the league game ended.

(3) Coach's evaluation: One head coach and one assistant coach from each of the 4 teams who participated in the Kanto university men's volleyball league game in the spring of year 2010 were collected. The total number of coaches was 8.They judged whether players performed well or not. Then, each player was placed into one of the following 2 groups; Wellperforming group (WPG) and Non well-performing group (NPG) based on the above-mentioned criteria.

\section{Results and Discussions}

\subsection{Relationship between state anxiety and success rate in game performance}

The relationship between the state anxiety scores and success rate in game performance was examined using correlation analysis. The results showed that there was negative correlation between the state anxiety score and success rate of spike on the day of game $(\mathrm{p}<.05)$, and there was positive correlation between the state anxiety score and success rate of reception in 3 time periods before the game $(p<.01)$. On the other hand, there was no correlation between the state anxiety score and success rate of block before the game.

Therefore, it is important to manage the state anxiety for keeping a high success rate in game performance, such as the success rate of spike and success rate of reception.
Table. 1

Correlation between state anxiety and success rate

\begin{tabular}{llcc}
\hline \multicolumn{1}{c}{ Time period } & Spike & Reception & Block \\
\hline The day before & -.31 & $.61^{* *}$ & .16 \\
The day of game & $-.40^{*}$ & $.63^{* *}$ & .19 \\
Just before the game & -.31 & $.41^{* *}$ & -.10 \\
Just after the game & $-.45^{* *}$ & .15 & $-.37 *$
\end{tabular}

$* \hookleftarrow \mathrm{p}^{\hookrightarrow} .05 * * \hookleftarrow \mathrm{p} \hookrightarrow .01$

\subsection{Relationship between state anxiety and coach's evaluation}

The comparison in state anxiety scores between each of the 4 times in WPG and NPG was examined using the unpaired t-test. The results showed that WPG scored lower than NPG in the score of state anxiety on the day of game and just after the game (see Fig.1).

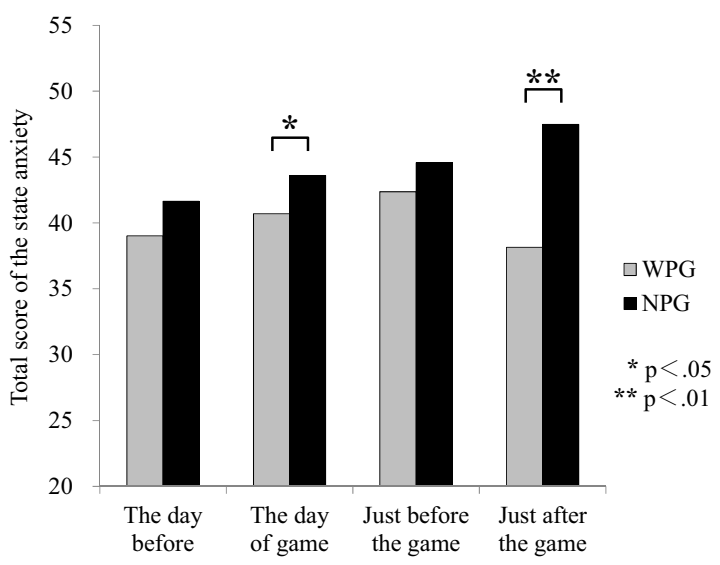

Fig. 1 Comparison in state anxiety scores between 2 groups

The comparison in temporal change of the score of state anxiety between the 4 times in each WPG and NPG was examined using the paired one-way analysis of variance. The result showed that WPG decreased the state anxiety score from just before the game to just after the game, but such temporal change was not shown in NPG (see Fig.2). 


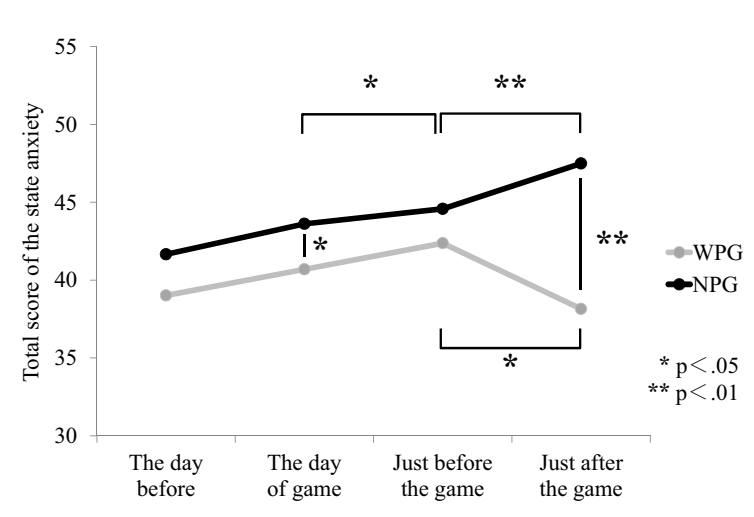

Fig. 2 Comparison in temporal change of the score of state anxiety between 4 times

Therefore, it was shown that well-evaluated players have low state anxiety before a game, and their state anxiety was diminished after the game, compared with badly-evaluated players.

\section{Conclusions}

The following two conclusions were obtained in this study:

(1) Relationships between state anxiety and success rate in game performance (spike, reception, block) showed different correlation depending on each game performance before the game.

(2) The group of players who performed well in games were collected by coaches and scored lower than the more badly-performing group in the score of state anxiety on the day of game and just after the game.

(3) Temporal change of the score of state anxiety from just before the game to just after the game was different depending on whether players performed well or not in games.

\section{References}

[1] K. Hamano et al., The Relationships between State Anxiety under Pre- and Post-Games and Athletic Performance in Volleyball Players on International Games, Journal of Health and Sports Science Juntendo University 4 (2000), 68-75. (in Japanese)

[2] N. Nakajima et al., The Relationship between State Anxiety under Pre-and Post-Games and Athletic Performance, Journal of Health and Sports Science Juntendo University 1 (1997), 26-35. (in Japanese)

[3] H, Shimizu et al., STATE-TRAIT ANXIETY INVENTORY no Nihongoban (Daigakuseiyou) no Sakusei. [Development of a Japanese Version (for University Students) of STATE-TRAIT ANXIETY INVENTORY], Japanese Journal of Educational Psychology 29-4 (1981), 62-67. (in Japanese)

[4] D. C. Spielberger, L. R. Gorsuch, \& F. R. Lushene, Manual for the state-trait anxiety inventory, Consulting Psychologists Press, California, 1970. 